\title{
Glycogen Supercompensation in the Rat Brain After Acute Hypoglycemia is Independent of Glucose Levels During Recovery
}

\author{
João M. N. Duarte ${ }^{1}$ Florence D. Morgenthaler ${ }^{2} \cdot$ Rolf Gruetter $^{1,3,4}$
}

Received: 20 September 2016 / Revised: 4 January 2017 / Accepted: 5 January 2017 / Published online: 12 January 2017

(C) Springer Science+Business Media New York 2017

\begin{abstract}
Patients with diabetes display a progressive decay in the physiological counter-regulatory response to hypoglycemia, resulting in hypoglycemia unawareness. The mechanism through which the brain adapts to hypoglycemia may involve brain glycogen. We tested the hypothesis that brain glycogen supercompensation following hypoglycemia depends on blood glucose levels during recovery. Conscious rats were submitted to hypoglycemia of $2 \mathrm{mmol} / \mathrm{L}$ for $90 \mathrm{~min}$ and allowed to recover at different glycemia, controlled by means of i.v. glucose infusion. Brain glycogen concentration was elevated above control levels after $24 \mathrm{~h}$ of recovery in the cortex, hippocampus and striatum. This glycogen supercompensation was independent of blood glucose levels in the post-hypoglycemia period. In the absence of a preceding hypoglycemia insult, brain glycogen concentrations were unaltered after $24 \mathrm{~h}$ under hyperglycemia. In the hypothalamus, which controls peripheral glucose homeostasis, glycogen levels were unaltered. Overall, we conclude that post-hypoglycemia glycogen supercompensation occurs in several brain areas and its magnitude is independent of plasma glucose levels. By supporting brain metabolism during recurrent hypoglycemia
\end{abstract}

João M. N. Duarte

joao.duarte@epfl.ch

1 Laboratory for Functional and Metabolic Imaging (LIFMET), Ecole Polytechnique Fédérale de Lausanne (EPFL), Station 6, 1015 Lausanne, Switzerland

2 Cellular Imaging Facility, University of Lausanne, Lausanne, Switzerland

3 Department of Radiology, University of Lausanne, Lausanne, Switzerland

4 Department of Radiology, University of Geneva, Geneva, Switzerland periods, glycogen may have a role in the development of hypoglycemia unawareness.

Keywords Glycogen · Brain · Hypoglycemia unawareness $\cdot$ Diabetes

\section{Introduction}

Episodes of hypoglycemia are a major complication of glycemia control by insulin administration. Diminished brain function during a hypoglycemia episode leads to potential physical danger and may have long-lasting damaging effects on the brain, resulting in cognitive impairment [1]. Moreover, recurrent hypoglycemia also impairs the body's defence mechanisms against hypoglycemia. Many patients with diabetes display a progressive decay in the counterregulatory response over time, resulting in reduction of hypoglycemia awareness [2], where the glycemia threshold for counter-regulation is shifted to lower plasma glucose levels and may be detected only upon severe neuroglycopenia [3].

The precise mechanisms that sense blood glucose and link the sensory machinery to the autonomic and endocrine responses which alert the patient to the low blood glucose levels and ultimately restore glucose homeostasis are not completely understood [4]. Much less is known about the mechanisms responsible for hypoglycemia unawareness, where a drop in glycemia is only detected when neuroglycopenia occurs [3]. It has been proposed that recurrent hypoglycemia leads to increased fuel availability to the brain, either glucose [5-7] or monocarboxylates [8] that are readily available from circulation. In addition, glycogen "supercompensation" may play a role in hypoglycemia unawareness, in which the brain adapts to hypoglycemia by 
increasing glucose storage in the form of extra glycogen content $[9,10]$.

Brain glycogen is hydrolysed to support energy metabolism and aid preserving neuronal function during hypoglycaemia [9-15]. Evidence supporting this post-hypoglycemia supercompensation has been reported in the human $[12,16]$, rat [9, 14], mouse [10] and rainbow trout [15]. However, the re-establishment of glycogen content in the brain after hypoglycaemia has been involved in controversy: (1) not all studies confirmed glycogen supercompensation after acute hypoglycaemia [11], (2) there have been reports suggesting that repeated hypoglycaemia does not result in increased glycogen levels [11, 16, 17], and (3) patients with type 1 diabetes and hypoglycaemia unawareness display brain glycogen levels similar to those in healthy subjects [18].

The aim of the present study was to test the hypothesis that brain glycogen concentrations are replenished above the normal levels after a hypoglycemia insult and to what extent this supercompensation is related to post-hypoglycemia glucose levels.

\section{Methods}

\section{Animals}

All procedures were performed in accordance to the Swiss animal welfare legislation under approval of the local ethics committee (EXPANIM-SCAV). Male Sprague-Dawley rats $(238 \pm 8 \mathrm{~g}, \mathrm{n}=22$, from Charles River, France) were housed on a 12-h light-dark cycle, room temperature at $22{ }^{\circ} \mathrm{C}$, humidity at $60 \%$, and with Food (Kliba Nafag 3800, Provimi-Kliba, Kaiseraugst, Switzerland) and water ad libitum.

\section{Intra-venous Catheter Implantation}

Micro-renathane catheters (inner/outer diameter in $\mathrm{mm}$ : 0.635/1.016; Braintree Scientific, Braintree, MA USA) were washed with $70 \%$ ethanol and soaked in saline with 200 U/mL heparin (Liquemin, Drossapharm, Basel, Switzerland) for $2 \mathrm{~h}$. After fasting for $6 \mathrm{~h}$, rats were anesthetized with $2 \%$ isoflurane (Attane, Minrad, NY, USA) in $30 \%$ oxygen in air. Surgery was performed under aseptic conditions. Core temperature was maintained at $37^{\circ} \mathrm{C}$. Areas in the right shoulder at the base of the neck and in the nape of the neck were shaved and thoroughly cleaned with $70 \%$ ethanol prior to skin incision. The right external jugular vein was isolated and a heparinised catheter was inserted and advanced to reach the vena cava. After secured to the vein with threads, the catheter was then tunnelled under the skin and exteriorised through the incision in the nape of the neck. Skin incisions were closed, catheter was filled with heparin solution $(200 \mathrm{U} / \mathrm{mL}$ in saline $)$ and rats were allowed to recover in a cage with water ad libitum. Food was provided on the following day. The catheter was flushed daily with saline and refilled with heparin solution under short isoflurane anesthesia. For three days, rats received $50 \mathrm{mg} / \mathrm{kg}$ intramuscular amoxicillin (Clamoxyl, Pfizer, Zurich, Switzerland) and $50 \mu \mathrm{g} / \mathrm{kg}$ subcutaneous buprenorphine (Temgesic, ESSEX Chemie, Luzern, Switzerland).

\section{Hypoglycemia and Recovery}

On the fifth day after surgery at 9:00 in the morning, $2 \mathrm{~h}$ after the beginning of the light cycle, rats were briefly anesthetised under isoflurane, and the implanted catheter was flushed and connected to an infusion line running through a swivel mounted in a counterbalanced lever arm (Instech, Plymouth Meeting, PA, USA). The rat was allowed to wake up in the cage with free access to water, and saline infusion started. The implanted catheter was also used for repeated blood sampling. When glucose was present in the infusate, infusion was interrupted and catheters were flushed before sampling, thus preventing contamination of blood samples with glucose from the infusate. In some cases, glycemia was confirmed by cross-measuring a drop of blood from the tail tip with the Ascencia Contour glucometer (Bayer, Zürich, Switzerland). Plasma glucose and lactate concentrations were quantified by the glucose and lactate oxidase methods, respectively, using GM7 Micro-Stat analysers (Analox Instruments, London, UK). Insulin (Huminsulin, Eli Lilly, Switzerland) was given as a bolus of $1 \mathrm{U} / \mathrm{kg}$ and continuous infusion at $6 \mathrm{U} / \mathrm{kg} / \mathrm{h}$ aiming at a target hypoglycemia of $2 \mathrm{mmol} / \mathrm{L}$ for $90 \mathrm{~min}$. Simultaneous glucose infusion at $50 \mu \mathrm{mol} / \mathrm{h}$ (using a $5 \mathrm{mmol} / \mathrm{L}$ solution) allowed controlling the degree of hypoglycemia. Insulin was only administered to rats submitted to hypoglycemia. Recovery from hypoglycemia was achieved by infusing glucose (5 or $20 \%$ in saline) with variable rate aiming at maintaining stable plasma glucose for $24 \mathrm{~h}$. The 12-h light-dark cycle was not interrupted. Control rats were submitted to either normo- or hyperglycemia for $24 \mathrm{~h}$ without a precedent hypoglycemia period. The following experimental groups were studied: control $(\mathrm{n}=6)$, hypoglycemia for $90 \mathrm{~min}$ $(n=3)$, normoglycemia followed by hyperglycemia $(n=4)$, and hypoglycemia followed by either normoglycemia $(n=4)$ or hyperglycemia $(n=5)$.

\section{Glycogen Quantification}

Rats were sacrificed using a focused microwave fixation device (Gerling Applied Engineering, Modesto, CA, USA) at $4 \mathrm{~kW}$ and $2450 \mathrm{MHz}$ for $2 \mathrm{~s}$ under short isoflurane 
anaesthesia [19]. Small tissue samples (10-25 mg) from occipital cortex, hippocampus, striatum and hypothalamus were dissected, frozen in liquid $\mathrm{N}_{2}$ and stored at $-80^{\circ} \mathrm{C}$. Samples were homogenised in $30 \mathrm{mmol} / \mathrm{L} \mathrm{HCl}$ with an ultrasonic homogenizer Sonopuls HD 2070 with the microtip MS 73 (Bandelin, Berlin, Germany) and, together with glycogen standards (concentrations from $2 \mu \mathrm{mol} / \mathrm{L}$ to $200 \mathrm{mmol} / \mathrm{L}$; glycogen from oyster Type II, Sigma-Aldrich, Basel, Switzerland), were boiled for $45 \mathrm{~min}$ at $90^{\circ} \mathrm{C}$. After adjusting $\mathrm{pH}$ to 5 with sodium acetate, samples and standards were incubated in the presence or absence of $20 \mathrm{ng} / \mathrm{mL}$ amylo- $\alpha-1,4-\alpha-1,6$-glucosidase (Roche Applied Science, Rotkreuz, Switzerland) for $2 \mathrm{~h}$ at $37^{\circ} \mathrm{C}$ [19]. After enzymatic digestion, $\mathrm{pH}$ was adjusted to 7 . After centrifugation at $14,000 \times g$ for $15 \mathrm{~min}$, supernatants were collected for glucose determination. Glucose concentration was determined by the glucose oxidase method coupled to a peroxidase reaction [20]. Briefly, samples, glycogen standards and freshly prepared glucose standards (concentrations from $1 \mu \mathrm{mol} / \mathrm{L}$ to $2 \mathrm{mmol} / \mathrm{L}$ ) were diluted in sodium phosphate buffer $(50 \mathrm{mmol} / \mathrm{L}, \mathrm{pH} 7.4)$ and incubated in 96 wells microplates with $2 \mathrm{U} / \mathrm{mL}$ glucose oxidase (Sigma-Aldrich), $0.2 \mathrm{U} / \mathrm{mL}$ horseradish peroxidase (Sigma-Aldrich) and $100 \mu \mathrm{mol} / \mathrm{L}$ AmplexRed (Invitrogen, Basel, Switzerland) for $30 \mathrm{~min}$ in the dark. The product of AmplexRed oxidation, resorufin, was detected at $560 \mathrm{~nm}$ with a PowerWave XS microplate spectrophotometer (BioTek, Luzern, Switzerland). From the glycogen standards submitted to the same extraction protocol as the tissue homogenates, calculated recovery was always above $96 \%$. Glycogen concentration was reported in terms of glucosyl equivalents released after amylo- $\alpha-1,4-\alpha-1,6$-glucosidase digestion, and normalised to tissue's wet weight.

\section{Statistical Analysis}

Data are shown as mean \pm SEM, and were analysed with ANOVA followed by Bonferroni multiple comparison tests, using Prism 5.04 (GraphPad, San Diego, CA, USA). Significance was considered for $\mathrm{P}<0.05$.

\section{Results}

To allow infusion and blood sample collection in freelymoving conscious rats at low blood pressure under severe hypoglycemia, a permanent catheter was placed into the superior vena cava. Good recovery from surgery was indicated by absence of weight loss within 5 days of recovery ( $238 \pm 8 \mathrm{~g}$ prior to surgery vs. $246 \pm 9 \mathrm{~g} 5$ days later).

Insulin was infused to achieve hypoglycaemia, which was maintained at $2 \mathrm{mmol} / \mathrm{L}$ or below during $99 \pm 6 \mathrm{~min}$ ( $\mathrm{n}=12$, Fig. 1). After hypoglycemia, rats were infused with glucose solutions to achieve normo- and hyperglycemia clamps for $24 \mathrm{~h}$, resulting in mean plasma glucose of $9.5 \pm 1.3 \mathrm{mmol} / \mathrm{L}(\mathrm{n}=4)$ and $18.2 \pm 1.5 \mathrm{mmol} / \mathrm{L}(\mathrm{n}=5)$, respectively. The animals subjected to the 24-h hyperglycemia clamp without preceding hypoglycaemia had mean plasma glucose of $18.9 \pm 2.2 \mathrm{mmol} / \mathrm{L}(\mathrm{n}=4)$. Plasma levels of lactate followed glycemia variations (Fig. 1). Brain glucose concentration was reduced by hypoglycemia $(\mathrm{P}<0.05$ for all regions), and increased in accordance to plasma glucose levels (Fig. 2a). The relation of brain glucose to plasma glucose, which depends on both glucose uptake and the cerebral metabolic rate of glucose $\left(\mathrm{CMR}_{\mathrm{glc}}\right)$ was similar among the experimental groups (Fig. 2b).
Fig. 1 Plasma glucose and lactate levels during insulininduced hypoglycemia and recovery by glucose infusion. Data are shown as mean \pm SEM. Experimental groups: control (Ctrl, black circles, $\mathrm{n}=6$ ), hypoglycemia (Hypo, white squares, $\mathrm{n}=3$ ), normoglycemia followed by hyperglycemia (Normo+Hyper, white circles, $\mathrm{n}=4$ ), and hypoglycemia followed by either normoglycemia (Hypo+Normo, white triangles, $\mathrm{n}=4$ ) or hyperglycemia (Hypo+Hyper, black triangles, $\mathrm{n}=5$ )
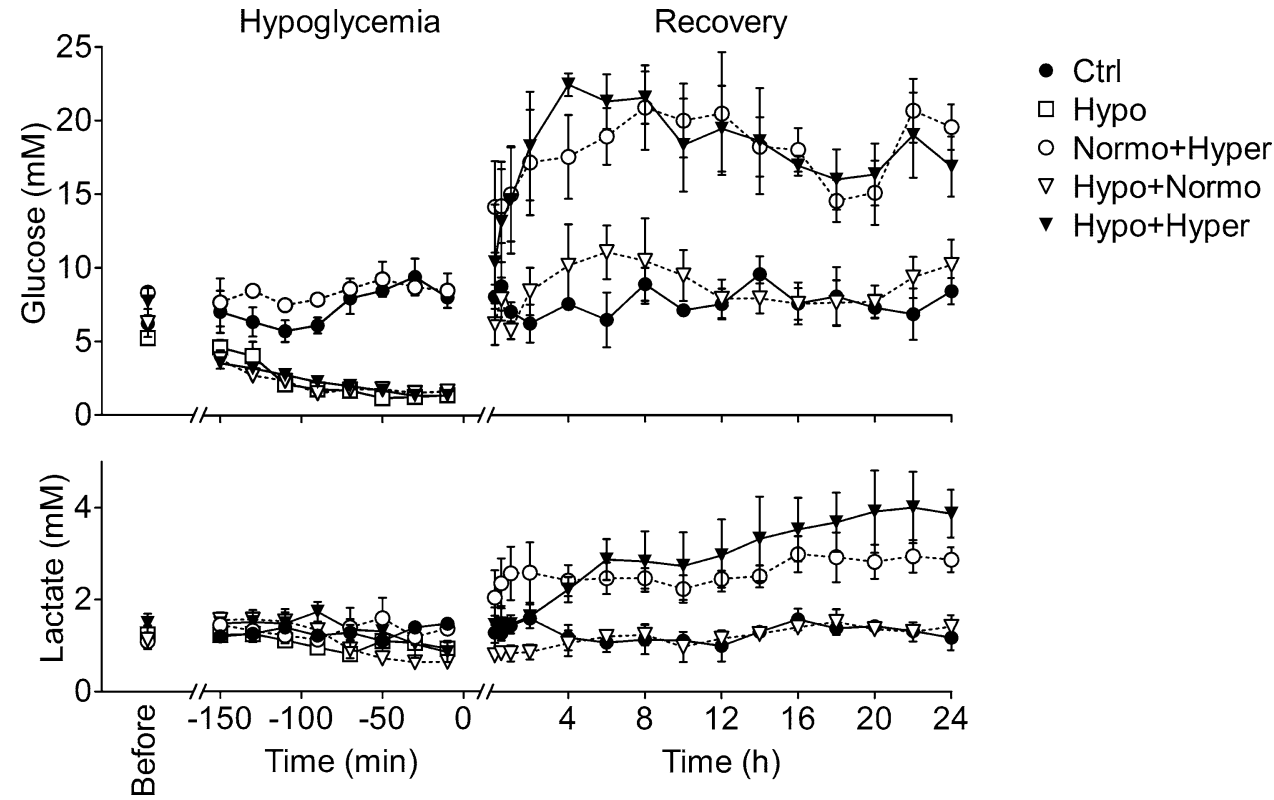
a

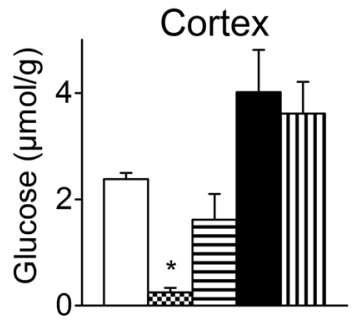

b

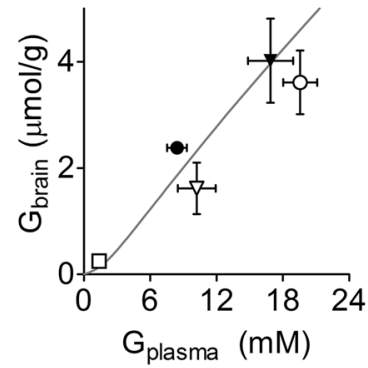

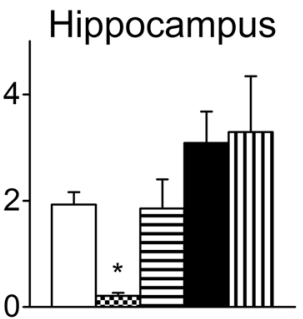

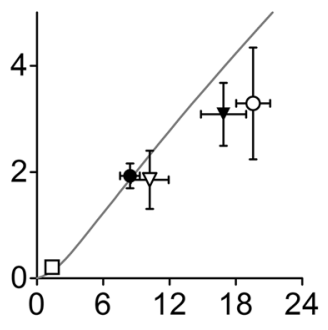

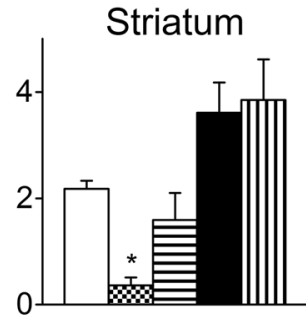

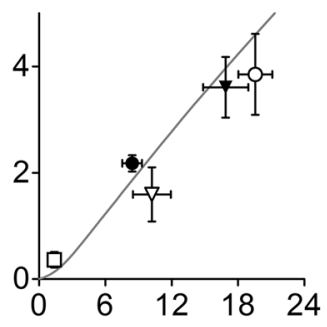

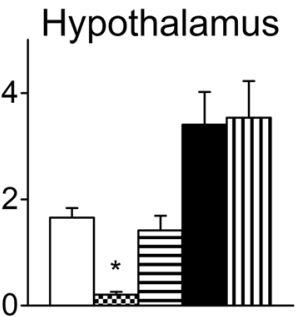

Ctrl

圈 Hypo

目 Hypo+Normo

Hypo+Hyper

向 Normo+Hyper

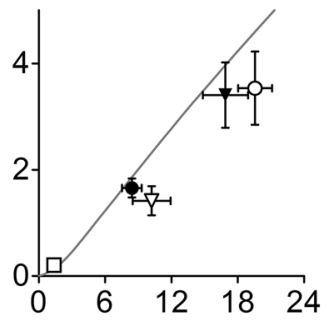

- Ctrl

- Hypo

o Normo+Hyper

$\nabla$ Hypo+Normo

v Hypo+Hyper

Fig. 2 Brain glucose concentration at the time of tissue collection (a), and its relation to plasma glucose levels (b). The grey line in panel $B$ depicts the relation of brain $\left(\mathrm{G}_{\text {brain }}\right)$ to plasma $\left(\mathrm{G}_{\text {plasma }}\right)$ glucose at steady-state in the rat brain under light $\alpha$-chloralose anesthesia reported previously [30]. Data are shown as mean \pm SEM of $n=3-6$, and were analysed with two-way ANOVA followed by Bonferroni post-tests. Glycemia challenges impacted brain glucose concentration $(\mathrm{F}=48.5, \mathrm{P}<0.0001)$. Brain areas show similar glucose levels
$(\mathrm{F}=0.74, \mathrm{P}>0.05)$, and interaction was not significant between the two variables $(\mathrm{F}=1.2, \mathrm{P}>0.05)$. Post-test comparison to control: $* \mathrm{P}<0.05$. Experimental groups: control $(\mathrm{Ctrl}$, black circles, $\mathrm{n}=6)$, hypoglycemia (Hypo, white squares, $\mathrm{n}=3$ ), normoglycemia followed by hyperglycemia (Normo+Hyper, white circles, $\mathrm{n}=4$ ), and hypoglycemia followed by either normoglycemia (Hypo+Normo, white triangles, $\mathrm{n}=4$ ) or hyperglycemia (Hypo+Hyper, black triangles, $\mathrm{n}=5$ )

$(\mathrm{P}<0.01, \mathrm{n}=3)$, compared to controls. During this period of hypoglycemia, glycogenolysis was estimated to be 0.03 , $0.04,0.02,0.04 \mu \mathrm{mol} / \mathrm{g} / \mathrm{min}$ in the cortex, hippocampus, striatum and hypothalamus, respectively, in agreement with previous reports [9]. A 24-h period of recovery caused a reversion of hypoglycemia-induced glycogen depletion in the cortex, hippocampus and striatum (Fig. 3). After $24 \mathrm{~h}$ of recovery under normoglycemia, rats displayed glycogen content increase in the cortex $(116 \pm 12 \%, \mathrm{P}<0.001, \mathrm{n}=4)$, hippocampus $(86 \pm 31 \%, \mathrm{P}<0.001, \mathrm{n}=4)$ and striatum $(96 \pm 37 \%, \mathrm{P}<0.05, \mathrm{n}=4)$, relative to controls. Recovery under hyperglycemia caused brain glycogen to rise above
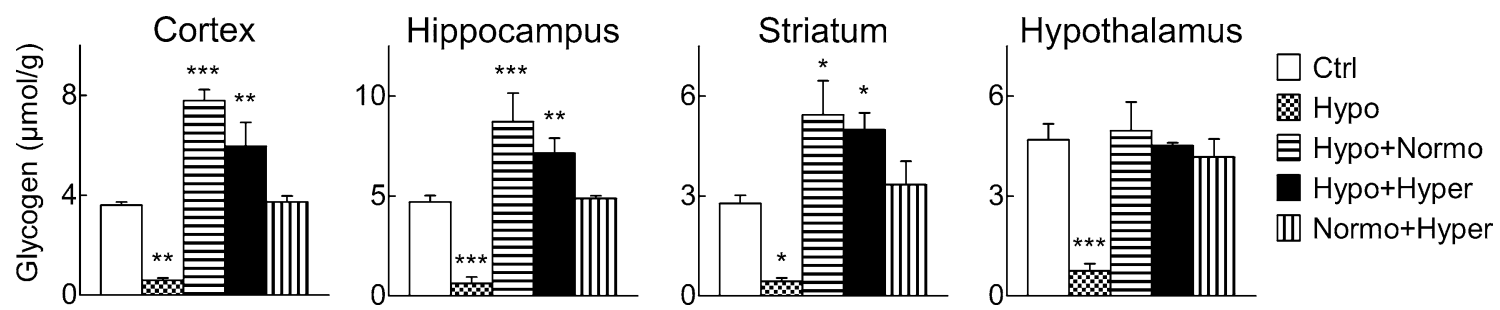

Fig. 3 Brain glycogen concentration (in glucosyl units) was increased after $24 \mathrm{~h}$ of recovery from $90 \mathrm{~min}$ of hypoglycemia in the cortex, hippocampus and striatum but not hypothalamus. Data are shown as mean \pm SEM of $n=3-6$, and were analysed with two-way ANOVA followed by Bonferroni post-tests. Both glycemia challenges and regional distribution were source of variation for glycogen concentration with F-values of $47.3(\mathrm{P}<0.0001)$ and $8.0(\mathrm{P}<0.001)$, respectively. Interaction was not significant between the two variables $(\mathrm{F}=1.8, \mathrm{P}>0.05)$. Post-test comparison to control: $* \mathrm{P}<0.05$, $* * \mathrm{P}<0.01, * * * \mathrm{P}<0.001$. Experimental groups: control $(\mathrm{Ctrl}, \mathrm{n}=6)$, hypoglycemia (Hypo, $n=3$ ), normoglycemia followed by hyperglycemia (Normo+Hyper, $n=4$ ), and hypoglycemia followed by either normoglycemia (Hypo+Normo, $\mathrm{n}=4$ ) or hyperglycemia (Hypo+Hyper, $\mathrm{n}=5$ ) 
controls by $65 \pm 26 \%(\mathrm{P}<0.01, \mathrm{n}=5), 52 \pm 16 \%(\mathrm{P}<0.01$, $\mathrm{n}=5)$ and $80 \pm 18 \%(\mathrm{P}<0.05, \mathrm{n}=5)$ in the cortex, hippocampus and striatum, respectively. In the hypothalamus, glycogen concentration recovered to baseline, i.e. not higher to that of controls, independently of blood glucose levels after the hypoglycemia episode. The hyperglycemia clamp without preceding hypoglycaemia was devoid of effects on brain glycogen (Fig. 3).

\section{Discussion}

The present study shows that excessive glycogen storage occurs in the rat brain after a severe hypoglycemia insult and is independent of the blood glucose levels during recovery. Notably, glycogen supercompensation upon 24-h recovery following hypoglycemia was observed in the hippocampus, cortex and striatum, but not in the hypothalamus.

Brain glycogen supercompensation after episodes of hypoglycemia have been matter of debate [9-12, 16-18, 23], probably due to distinct experimental approaches, hypoglycemia severity and animal models used. Using ${ }^{13} \mathrm{C}$ magnetic resonance spectroscopy in rats to measure brain glycogen upon labelling from $\left[1{ }^{13} \mathrm{C}\right]$ glucose, Choi et al. reported a $153 \%$ increase in the amount of ${ }^{13} \mathrm{C}$-labeled glycogen after 7 -h hyperglycemia recovery from insulin-induced acute hypoglycemia of $2 \mathrm{mmol} / \mathrm{L}$ for $2 \mathrm{~h}$, when compared to the concentration measured before hypoglycemia [9]. In mice, after 6-h of euglycemic recovery from insulin-induced acute hypoglycemia there was a $25 \%$ increase in whole brain glycogen content, relative to that measured in controls [10]. However, $27 \mathrm{~h}$ after the hypoglycemia period, brain glycogen levels approached baseline levels. Furthermore, the authors [10] observed similar results when animals had been previously submitted to daily recurrent hypoglycemia insults for 9 days. Resembling the effect of systemic hypoglycemia, neuroglycopenia induced by repetitive intracerebroventricular injection of 2-deoxy-D-glucose for 3 days induced increases in brain glycogen content in the hypothalamus and cortex by 69 and $153 \%$, respectively, in comparison to saline-injected rats [23]. In contrast to these reports, Herzog et al. [11] did not find glycogen supercompensation after either acute or recurrent hypoglycemia in the rat cortex, cerebellum or and hypothalamus. Moreover, brain glycogen turnover in rats recovering from chronic hypoglycemia $(<3 \mathrm{mmol} / \mathrm{L}$ for 12-14 days) was similar to controls [17]. Oz et al. [18] used ${ }^{13} \mathrm{C}$ magnetic resonance spectroscopy in conjunction with $\left[1-{ }^{13} \mathrm{C}\right]$ glucose administration measure brain glycogen content and metabolism in patients with type 1 diabetes and hypoglycemia unawareness, as well as healthy volunteers. This study also did not support the hypothesis that post-hypoglycemia glycogen supercompensation is the driver for hypoglycaemia unawareness [18], although a single hypoglycaemia episode was shown to elicit glycogen supercompensation in healthy subjects $[12,16]$. Nevertheless, increased brain glucose storage could be favoured by increased glucose uptake after hypoglycaemia. Indeed, it has been proposed that recurrent hypoglycemia leads to a sustained increase in brain glucose [5-7, 17] due to enhanced expression and density of glucose carriers [24, 25].

Brain glycogen supercompensation was also observed in rats after depletion upon exhaustive exercise [26]. While brain glycogen levels decreased with exercise, they were supercompensated at $6 \mathrm{~h}$ after exercise in several brain areas, including the cortex, hippocampus and hypothalamus [26]. The authors further observed that brain glycogen supercompensation after exercise positively correlated with the decrease rate during exercise and that it was sustained until $24 \mathrm{~h}$ after exercise in both cortex and hippocampus (long-lasting supercompensation) but not in the hypothalamus, a brain area involved in maintenance of peripheral glucose homeostasis. In analogy to the present study, one may speculate that overshot of glycogen content may also occur in the hypothalamus after a few hours of recovery from hypoglycemia but at $24 \mathrm{~h}$ they had already normalised. Accordingly, repetitive neuroglucopenia in three consecutive days induced much larger glycogen supercompensation in cortex than hypothalamus [23]. Increased glial glycogen stores can eventually sustain neuronal activity for a longer period [13] during a subsequent hypoglycemia episode and, therefore, contribute to the shift in the glycemia threshold at which counter-regulation initiates. However, it remains to be addressed whether excessive content of glycogen can effectively buffer brain glucose supply to glucose-sensing neurons for longer periods.

In our study, glycogenolysis during hypoglycaemia was estimated to range between 0.02 and $0.04 \mu \mathrm{mol} / \mathrm{g} / \mathrm{min}$ across the four measured regions, which is a small contribution to the brain's energy requirements. Relative to the respective regional $\mathrm{CMR}_{\text {glc }}$ in awake rats [27, 28], we estimated that glycogenolysis only accounted to about $2 \%$ in the striatum, $3 \%$ in the cortex, $5 \%$ in the hippocampus, and $7 \%$ in the hypothalamus. However, blood-to-brain glucose transport becomes limiting for hexokinase at hypoglycaemia, resulting in reduced $\mathrm{CMR}_{\text {glc }}[29,30]$. Therefore, with a 50\% reduction in $\mathrm{CMR}_{\text {glc }}$ during hypoglycemia [29], glycogenolysis may represent up to $30 \%$ of glucose utilisation. In addition, oxidation of endogenous substrates such as amino acids can also contribute to fuel the brain during hypoglycaemia, and their normal concentration is readily re-established upon recovery [31,32], at a rate much faster than the replenishment of brain glycogen stores [31]. 
Although this study was not designed to investigate glucose transport and consumption rates, we compared brain versus plasma glucose levels with the expected values that we derived from a previous study [30] (Fig. 2b). Care should be taken when comparing these datasets because arterial plasma glucose was measured in our previous study [30], and we now measured glucose in venous plasma collected from the vena cava. Since venous plasma has lower glucose levels than arterial plasma, one should expect a displacement of the present data from the line plotted in Fig. $2 b$ towards larger brain to plasma glucose. On the other hand, awake rats have larger $\mathrm{CMR}_{\mathrm{glc}}$ and thus lower brain glucose levels at the same glycemia, when compared to anesthetised rats (in previous study [30]). In spite of that, such differences between the two datasets are likely not detectable by this approach. It is interesting to note that the relation between brain and plasma glucose, which is dependent on glucose uptake and $\mathrm{CMR}_{\mathrm{glc}}$, in rats submitted to hypoglycemia and subsequent recovery for $24 \mathrm{~h}$ is not substantially different from that previously reported at steady-state in control rats under light $\alpha$-chloralose anesthesia [30]. In other words, we did not observe important deviations of measured brain glucose levels from the expected values at a given plasma glucose. Acute hypoglycemia of $2 \mathrm{mmol} / \mathrm{L}$ was reported to cause a $40-50 \%$ reduction of $\mathrm{CMR}_{\mathrm{glc}}$ in the four regions analysed in our study [29]. More severe hypoglycemia episodes (blood glucose of $1-1.5 \mathrm{mmol} / \mathrm{L}$ ) result in further reduction of $\mathrm{CMR}_{\mathrm{glc}}$ by nearly $75 \%$ [32]. However, $\mathrm{CMR}_{\mathrm{glc}}$ is rapidly normalised upon recovery (within $10 \mathrm{~min}$ in the absence of hypoglycemic coma) [32]. On the other hand, $\mathrm{CMR}_{\mathrm{glc}}$ is not expected to change upon acute hyperglycemia [33]. While it has been proposed that recurrent hypoglycemia leads to increased glucose transport from plasma to brain [5-7], the present results suggest that a single hypoglycemia episode does not alter the brain-to-blood glucose relationship. Therefore, assuming similar $\mathrm{CMR}_{\mathrm{glc}}$ for all experimental groups, glucose transport across the blood-brain-barrier does not appear contributing to augment brain glycogen storage after hypoglycemia.

All together, these results suggest that glycogen content and metabolism may have a role in diabetic hypoglycemia unawareness, even though very little is known on brain glycogen metabolism in diabetes. While animal models of diabetes show unaltered glucose transport at the blood-brainbarrier (see [34] and references therein), thus leading to high brain glucose concentration at hyperglycemia, they do not display evident alteration of brain glycogen levels (reviewed in [35]). Similarly, patients with insulin-dependent diabetes and hypoglycemia unawareness display normal brain glycogen concentration [18]. Contrastingly, increased glycogen concentration was reported in the brain of obese, insulin resistant Zucker diabetic fatty rats [36].
Insulin modulates brain function and was reported to act on brain glycogen [19, 37]. Insulin and insulin-like growth factor I (IGF-I) have been implicated in the regulation of glycogen synthesis in cultured astrocytes [37]. We also previously found that the administration of somatostatin to supress systemic insulin release, while infusing glucose, resulted in decreased glycogen content in the rat brain [19]. A limitation of this study is that control rats were not administered exogenous insulin. While an experimental group with insulin infusion and normoglycemic clamp was not included, the 24-h hyperglycemic clamp is known to result in hyperinsulinemia. Therefore, it is likely that all animal groups, including those not challenged with hypoglycaemia, had high circulating insulin levels. Moreover, brain glycogen supercompensation after exhaustive exercise was reported to be unrelated to blood insulin levels [26].

We conclude that brain glycogen concentrations are replenished above their normal levels after a single hypoglycemic insult independently of the post-hypoglycemia glucose levels. The effects of severity and duration of the hypoglycemia insult on the degree of glycogen supercompensation remain to be ascertained.

Acknowledgements This work was supported by the Swiss National Science Foundation (SNSF, Grant 122498), the National Institutes of Health (Grant R01NS042005), and the Centre d'Imagerie BioMédicale (CIBM) of the UNIL, UNIGE, HUG, CHUV, EPFL and the Leenaards and Jeantet Foundations. JMND was supported by the SNSF Ambizione program (Grant 148250). The authors are grateful to Dr. Igor Allaman (Laboratory of Neuroenergetics and Cellular Dynamics, EPFL) and Dr. Ana F. Soares (LIFMET, EPFL) for fruitful discussions.

\section{Compliance with Ethical Standards}

Conflict of interest None.

\section{References}

1. Warren RE, Frier BM (2005) Hypoglycemia and cognitive function. Diabetes Obes Metab 7:493-503

2. Cryer PE (2004) Diverse causes of hypoglycemia-associated autonomic failure in diabetes. N Engl J Med 350:2272-2279

3. Fanelli CG, Pampanelli S, Porcellati F, Bolli GB (1998) Shift of glycaemic thresholds for cognitive function in hypoglycemia unawareness in humans. Diabetologia 41:720-723

4. Marty N, Dallaporta M, Thorens B (2007) Brain glucose sensing, counterregulation, and energy homeostasis. Physiology 22:241-251

5. Boyle PJ, Nagy RJ, O'Connor AM, Kempers SF, Yeo RA, Qualls C (1994) Adaptation in brain glucose uptake following recurrent hypoglycemia. Proc Natl Acad Sci USA 91:9352-9356

6. Criego AB, Tkáč I, Kumar A, Thomas W, Gruetter R, Seaquist ER (2005) Brain glucose concentrations in patients with type 1 diabetes and hypoglycemia unawareness. J Neurosci Res 79:42-47 
7. McCall AL, Fixman LB, Fleming N, Tornheim K, Chick W, Ruderman NB (1986) Chronic hypoglycemia increases brain glucose transport. Am J Physiol Endocrinol Metab 251:E442-E447

8. Mason GF, Petersen KF, Lebon V, Rothman DL, Shulman GI (2006) Increased brain monocarboxylic acid transport and utilization in type 1 diabetes. Diabetes 55:929-934

9. Choi IY, Seaquist ER, Gruetter R (2003) Effect of hypoglycemia on brain glycogen metabolism in vivo. J Neurosci Res 72:25-32

10. Canada SE, Weaver SA, Sharpe SN, Pederson BA (2011) Brain glycogen supercompensation in the mouse after recovery from insulin-induced hypoglycemia. J Neurosci Res 89:585-591

11. Herzog RI, Chan O, Yu S, Dziura J, McNay EC, Sherwin RS (2008) Effect of acute and recurrent hypoglycemia on changes in brain glycogen concentration. Endocrinology 149:1499-1504

12. Oz G, Kumar A, Rao JP, Kodl CT, Chow L, Eberly LE, Seaquist ER (2009) Human brain glycogen metabolism during and after hypoglycemia. Diabetes 58:1978-1985

13. Suh SW, Bergher JP, Anderson CM, Treadway JL, Fosgerau K, Swanson RA (2007) Astrocyte glycogen sustains neuronal activity during hypoglycemia: studies with the glycogen phosphorylase inhibitor CP-316,819 ([R-R*,S*]-5-chloro-N-[2-hydroxy-3-(methoxymethylamino)-3-oxo-1-(phenylmethyl)propyl]1H-indole-2-carboxamide). J Pharmacol Exp Ther 321:45-50

14. Cardell M, Siesjö BK, Wieloch T (1991) Changes in pyruvate dehydrogenase complex activity during and following severe insulin-induced hypoglycemia. J Cereb Blood Flow Metab 11(1):122-128

15. Blanco AM, Gómez-Boronat M, Pérez-Maceira J, Mancebo MJ, Aldegunde M (2015) Brain glycogen supercompensation after different conditions of induced hypoglycemia and sustained swimming in rainbow trout (Oncorhynchus mykiss). Comp Biochem Physiol A Mol Integr Physiol 187:55-60

16. Öz G, DiNuzzo M, Kumar A, Moheet A, Khowaja A, Kubisiak K, Eberly LE, Seaquist ER (2016) Cerebral glycogen in humans following acute and recurrent hypoglycemia: implications on a role in hypoglycemia unawareness. J Cereb Blood Flow Metab. doi: $10.1177 / 0271678 X 16678240$

17. Lei H, Gruetter R (2006) Effect of chronic hypoglycemia on glucose concentration and glycogen content in rat brain: a localized ${ }^{13}$ C NMR study. J Neurochem 99:260-268

18. Oz G, Tesfaye N, Kumar A, Deelchand DK, Eberly LE, Seaquist ER (2012) Brain glycogen content and metabolism in subjects with type 1 diabetes and hypoglycemia unawareness. J Cereb Blood Flow Metab 32(2):256-263

19. Morgenthaler FD, Koski DM, Kraftsik R, Henry PG, Gruetter R (2006) Biochemical quantification of total brain glycogen concentration in rats under different glycemic states. Neurochem Int 48:616-622

20. Poitry-Yamate C, Lei H, Gruetter R (2009) The rate-limiting step for glucose transport into the hypothalamus is across the bloodhypothalamus interface. J Neurochem 109:38-45

21. Cruz NF, Dienel GA (2002) High glycogen levels in brains of rats with minimal environmental stimuli: implications for metabolic contributions of working astrocytes. J Cereb Blood Flow Metab 22:1476-1489

22. Sagar SM, Sharp FR, Swanson RA (1987) The regional distribution of glycogen in rat brain fixed by microwave irradiation. Brain Res 417:172-174
23. Alquier T, Kawashima J, Tsuji Y, Kahn BB (2007) Role of hypothalamic adenosine 5'-monophosphate-activated protein kinase in the impaired counterregulatory response induced by repetitive neuroglucopenia. Endocrinology 148:1367-1375

24. Kumagai AK, Kang YS, Boado RJ, Pardridge WM (1995) Upregulation of blood-brain barrier GLUT1 glucose transporter protein and mRNA in experimental chronic hypoglycemia. Diabetes 44:1399-1404

25. Simpson IA, Appel NM, Hokari M, Oki J, Holman GD, Maher F, Koehler-Stec EM, Vannucci SJ, Smith QR (1999) Blood-brain barrier glucose transporter: effects of hypo- and hyperglycemia revisited. J Neurochem 72:238-247

26. Matsui $\mathrm{T}$, Ishikawa $\mathrm{T}$, Ito $\mathrm{H}$, Okamoto $\mathrm{M}$, Inoue $\mathrm{K}$, Lee $\mathrm{MC}$, Fujikawa T, Ichitani Y, Kawanaka K, Soya H (2012) Brain glycogen supercompensation following exhaustive exercise. J Physiol 590(Pt 3):607-616

27. Sokoloff L, Reivich M, Kennedy C, Des Rosiers MH, Patlak CS, Pettigrew KD, Sakurada O, Shinohara M (1977) The $\left[{ }^{14} \mathrm{C}\right]$ deoxyglucose method for the measurement of local cerebral glucose utilization: theory, procedure, and normal values in the conscious and anesthetized albino rat. J Neurochem 28(5):897-916

28. Archer DP, Elphinstone MG, Pappius HM (1990) The effect of pentobarbital and isoflurane on glucose metabolism in thermally injured rat brain. J Cereb Blood Flow Metab 10:624-630

29. Pelligrino DA, Segil LJ, Albrecht RF (1990) Brain glucose utilization and transport and cortical function in chronic vs. acute hypoglycemia. Am J Physiol 259(5 Pt 1):E729-E735

30. Duarte JMN, Gruetter R (2012) Characterization of cerebral glucose dynamics in vivo with a four-state conformational model of transport at the blood-brain-barrier. J Neurochem 121(3):396-406

31. Agardh CD, Folbergrová J, Siesjö BK (1978) Cerebral metabolic changes in profound, insulin-induced hypoglycemia, and in the recovery period following glucose administration. J Neurochem 31(5):1135-1142

32. Ghajar JB, Plum F, Duffy TE (1982) Cerebral oxidative metabolism and blood flow during acute hypoglycemia and recovery in unanesthetized rats. J Neurochem 38(2):397-409

33. Duckrow RB (1988) Glucose transfer into rat brain during acute and chronic hyperglycemia. Metab Brain Dis 3(3):201-209

34. Duarte JMN, Carvalho RA, Cunha RA, Gruetter R (2009) Caffeine consumption attenuates neurochemical modifications in the hippocampus of streptozotocin-induced diabetic rats. J Neurochem 111:368-379

35. Duarte JMN (2015) Metabolic alterations associated to brain dysfunction in diabetes. Aging Dis 6(5): 304-321

36. Sickmann HM, Waagepetersen HS, Schousboe A, Benie AJ, Bouman SD (2010) Obesity and type 2 diabetes in rats are associated with altered brain glycogen and amino-acid homeostasis. J Cereb Blood Flow Metab 30(8):1527-1537

37. Dringen R, Hamprecht B (1992) Glucose, insulin, and insulinlike growth factor I regulate the glycogen content of astrogliarich primary cultures. J Neurochem 58(2):511-517 